\title{
Research Paper \\ A Comparative Study on the Effects of Using Hydroalcoholic Extracts of Linum Usitatissimum and Rosa Damascena on Liver Function in Adult Male Rats
}

\author{
Mohammad Mehdi Padam ${ }^{1}$ (), *Ameneh Khoshvaghti² (1)
}

1. Faculty of Veterinary Medicine, Kazerun Branch, Islamic Azad University, Kazerun, Iran.

2. Department of Clinical Sciences, Faculty of Veterinary Medicine, Kazerun Branch, Islamic Azad University, Kazerun, Iran.

\begin{tabular}{|c|c|}
\hline $\begin{array}{l}\text { Use your device to scan } \\
\text { and read the article online }\end{array}$ & Citation Padam M, Khoshvaghti A. [A Comparative Study on the Effects of Using Hydroalcoholic Extracts of Linum Usitatis- \\
\hline 口75:日a & simum and Rosa Damascena on Liver Function in Adult Male Rats (Persian)]. Quarterly of "The Horizon of Medical Sciences". \\
\hline & 2020; 26(1):54-67. https://doi.org/10.32598/hms.26.1.3116.1 \\
\hline 낙다굴 & doi'https://doi.org/10.32598/hms.26.1.3116.1 \\
\hline
\end{tabular}

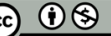

Received: 16 Apr 2019

Accepted: 24 Sep 2019

Available Online: 01 Jan 2020

Keywords:

Linum Usitatissimum Liver ,Rosa Dama-

scena, Rat

\section{A B STRACT}

Aims Damage to liver tissue and its dysfunction is very important and if left untreated, it can cause serious problems and even death. In this study, we aimed to investigate the effects of the hydroalcoholic extracts of Linum usitatissimum and Rosa damascena on liver enzymes, total protein, bilirubin, albumin, and serum glucose levels.

Materials and Methods This is a non-randomized clinical trial conducted on 42 male rats divided into 6 groups; control group (group 1) received only sufficient water and food, groups 1 and 2 received 300 and $500 \mathrm{mg} / \mathrm{kgB} . \mathrm{W}$ Linum usitatissimum extract, groups 3 and 4 received 500 and $1000 \mathrm{mg} / \mathrm{kgB} . \mathrm{W}$ Rosa damascena, and group 6 received 100 mg/ kgB.W Linum usitatissimum plus 250 mg/ kgB.W Rosa damascena extracts intraperitoneally for 28 days. After the last injection, the rats were weighed and their blood samples were collected. The study parameters were measured using a colorimetric method by a spectrophotometer, and then were analyzed using ANOVA and Tukey's test in SPSS V. 25 at a significance level of $\mathrm{P}<0.05$.

Findings There was no significant difference between alanine aminotransferase, alkaline phosphatase, total and direct bilirubin levels in the control group in comparison with other groups $(P>0.05)$. In the groups received Rosa damascena extract, there was a significant difference between total protein and albumin levels compared to the control group $(\mathrm{P}<0.05)$. Moreover, there was a significant difference between serum glucose and aspartate aminotransferase in the control group compared to other groups $(P<0.05)$.

Conclusion Linum usitatissimum and Rosa damascena have no negative effect on the liver function. The probability of diarrhea occurrence and the possible effects on the total protein and serum albumin after using Rosa damascena, and the effects of different doses of Linum usitatissimum on the glucose levels should be taken into account.

\section{Extended Abstract}

\section{Introduction}

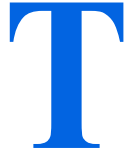

he liver is responsible for vital functions in the body. Liver disease and dysfunction cause significant damage to the body, and even if the disease becomes chronic in the liver and liver problems are not treated, it can lead to death. Liver diseases often have symptoms similar to those of other diseases, which can make it more difficult and slower to diagnose them [1].

The liver is the largest gland in the body and plays an important role in the body's metabolism and detoxification of various substances, so the health of this organ is important

\section{* Corresponding Author:}

\section{Ameneh khoshvaghti, PhD.}

Address: Department of Clinical Sciences, Faculty of Veterinary Medicine, Kazerun Branch, Islamic Azad University, Kazerun, Iran.

Tel: +98 (71) 42243936

E-mail: khoshvaghti2004@yahoo.com 
[2]. The liver, which plays an important role in the metabolism and storage of glucose as glycogen, plays a role in regulating blood glucose levels [7].

Albumin is the main plasma protein and its main function is to regulate the colloidal osmotic pressure [3]. Biliberin is one of the yellow bile pigments obtained from the breakdown of hemoglobin [4]. The use of medicinal plants has been common since ancient times. They have fewer side effects than chemical drugs, especially when common treatments are unable to control the disease. In recent years, attention to complementary therapies and therapeutic effects of natural compounds of plant origin has increased [5]. One of these plants is Rosa damascena from Rosaceae family [6]. Its main compounds are anthocyanins, cyanidin 3, 5-d-O-glucosides and several compounds such as camphor, quercetin, galactoside, arabinose, and ginnol. Its antioxidants can be helpful in preventing heart disease [8].

This plant is also used in the treatment of other diseases such as respiratory allergies and insomnia $[9,10,11]$. Another plant is Linum usitatissimum with bay leaves. Its seed is rich in various phenylpropanoids, especially lignans, flavonoids and cyanogenic glycosides. It also contains alphalinolenic acid and omega 3 [12]. It is used to treat many diseases such as irritable bowel syndrome [13, 14]. Since sometimes, these medicinal plants are overused regardless of their side effects or desired dose, in order to determine their effective dose and prevent liver damage by not interfering with liver factors, this study aimed to investigate the effective doses of the hydroalcoholic extracts of Linum usitatissimum and Rosa damascena on liver enzymes, total protein, bilirubin, albumin, and serum glucose in rats.

\section{Materials and Methods}

This is a non-randomized clinical trial conducted on 42 male Wistar rats in animal Laboratory of Islamic Azad University of Kazeroon branch in 2019. Rats were divided into 6 groups; control group (group 1) received only sufficient water and food, groups 1 and 2 received 300 and 500 $\mathrm{mg} / \mathrm{kgB} . \mathrm{W}$ Linum usitatissimum extract, groups 3 and 4 received 500 and $1000 \mathrm{mg} / \mathrm{kgB} . \mathrm{W}$ Rosa damascena, and group 6 received $100 \mathrm{mg} / \mathrm{kgB}$.W Linum usitatissimum plus $250 \mathrm{mg} / \mathrm{kgB}$.W Rosa damascena extracts intraperitoneally for 28 days. After the last injection, the rats were weighed and their blood samples were collected. The study parameters were measured using a colorimetric method by a spectrophotometer, and then were analyzed using ANOVA and Tukey's test in SPSS V. 25 at a significance level of $\mathrm{P}<0.05$.

\section{Results}

There was no significant difference between Alanine Aminotransferase (ALT), Alkaline Phosphatase (ALP), total and direct bilirubin levels in the control group in comparison with other groups $(\mathrm{P}>0.05)$. In the groups received Rosa damascena extract, there was a significant difference between total protein and albumin levels compared to the control group $(\mathrm{P}<0.05)$. Moreover, there was a significant difference between serum glucose and aspartate Aminotransferase (AST) in the control group compared to other groups $(\mathrm{P}<0.05)$.

\section{Discussion}

Consumption of the hydroalcoholic extract of Linum usitatissimum at a dose of $300 \mathrm{mg} / \mathrm{kg}$ increased the glucose levels and caused a significant statistical difference between the mean serum glucose levels of rats in this group and the control group $(\mathrm{P}<0.05)$. However, $500 \mathrm{mg} / \mathrm{kg}$ Linum usitatissimum reduced the glucose level and this difference was not significant (Table 1). Consumption of hydroalcoholic extract of Rosa damascena alone and in combination with Linum usitatissimum reduced the glucose level, but only the effect of $1000 \mathrm{mg} / \mathrm{kg}$ Rosa damascena extract was significant which caused a significant statistical difference between the mean serum glucose levels of rats in this group and other groups except with the group received $500 \mathrm{mg} / \mathrm{kg}$ Rosa damascena extract (Table 1).

Nikbakht and Gheitasi [15] reported that blood glucose levels were significantly reduced in consumers of hydroalcoholic extract of Citrullus colocynthis and Rosa damascena compared to controls. The effect of Rosa damascena extract on blood glucose level can be due to the presence of flavonoids and their glycosidic compounds. In one study, the effect of Rosa damascena methanolic extract in comparison with acarbose (an alpha-glucosidase inhibitor) in normal and diabetic rats was investigated and the strong inhibitory effect of alpha glycosidase was confirmed [16]. It seems that the effects of different amounts of glycosidic compounds and glycosides of different plants as well as the amount of glycosides and flavonoid compounds on the glucose level are different; hence, when using Linum usitatissimum extract, the dose of the extract should be determined based on the blood glucose level of the patient so that reducing or increasing glucose does not cause problems for the person. We can even use the proper dose of Linum usitatissimum extract to regulate serum glucose levels [17].

The effect of different doses of hydroalcoholic extracts of Linum usitatissimum and Rosa damascena on ALP level is similar to the effect of these extracts on the glucose level, 


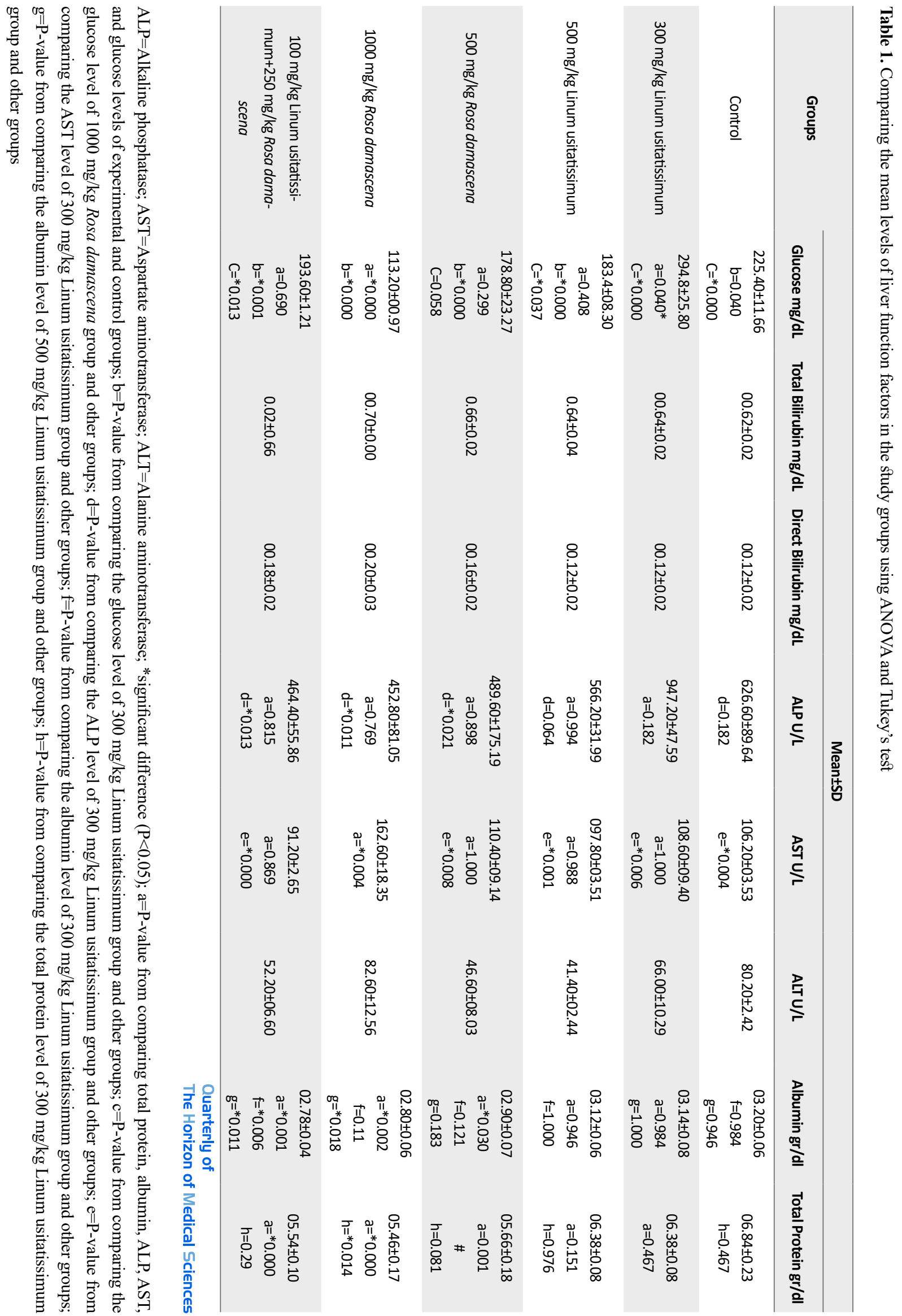


but to a lesser extent such that $300 \mathrm{mg} / \mathrm{kg}$ Linum usitatissimum hydroalcoholic extract increased the average activity of this enzyme; however, the use of other doses of extracts reduced the activity of ALP and the highest reduction was observed after using $1000 \mathrm{mg} / \mathrm{kg}$ Rosa damascena hydroalcoholic extract, although these changes are not large enough to cause a statistically significant difference between the mean level of this enzyme in these groups compared to the control group, but a statistically significant difference was observed between the mean levels of ALP in $300 \mathrm{mg} / \mathrm{kg} \mathrm{Li}$ num usitatissimum group, in the groups received different dose of Rosa damascena, and the group treated with both Linum usitatissimum and Rosa damascena.

This finding can be a warning to the consumers of Linum usitatissimum extract about the harmful effects of this extract on the liver, especially the bile ducts. In high doses of Linum usitatissimum extract and low and high doses of Rosa damascena extract and even the combination of the extracts of both plants, the antioxidant effect of flavonoids was predominant, such that the consumption of these extracts had no significant effect on the main indicators of a healthy liver (ALP, ALT, total and direct bilirubin) [18, 19].

The use of $1000 \mathrm{mg} / \mathrm{kg}$ Rosa damascena hydroalcoholic extract significantly increased the AST level. Given that ALT level, which is more specific for the diagnosis of liver disease than AST [20], has not changed and ALP level even decreased in this group, this increase in AST is most likely not due to a liver problems. If ensuring the absence of hemolysis in the tested samples, this increase can be due to muscle injury. In this case, the creatine phosphokinase enzyme level measurement can be very decisive.

The albumin and total protein levels in groups that consumed low and high doses of Rosa damascena extract and the combination of Rosa damascena and Linum usitatissimum extracts showed a significant decrease. Due to the severe diarrhea observed in these groups that consumed Rosa damascena, the decrease in albumin level can be due to its excretion through the gastrointestinal tract. There was a significant statistical difference between the mean levels of albumin and total protein in the groups received Rosa damascena and Linum usitatissimum hydroalcoholic extracts which was then due to the lack of excretion of total protein and albumin from their body.

The changes in albumin and total protein levels in these groups are not related to the process of protein synthesis in the liver. AKbari et al [21] in a study reported that Rosa damascena has the least nephrotoxic and hepatotoxic effects; however, its very high doses (1440 mg/kg body weight) can lead to hepatotoxic effects. Therefore, the hepatic effects of Rosa damascena have been considered to be dose-dependent [21].

\section{Conclusion}

Linum usitatissimum and Rosa damascena have no negative effect on the liver function. The probability of diarrhea occurrence and the possible effects on the total protein and serum albumin after using Rosa damascena, and the effects of different doses of Linum usitatissimum on the glucose levels should be taken into account.

\section{Ethical Considerations}

\section{Compliance with ethical guidelines}

All experiments in this study were according to guidelines of Animal Research Ethics Committee of Islamic Azad University of Kazeroon branch (Code: IR.IAU.KAU. REC.1398.048 ).

Funding

The present paper was extracted from the $\mathrm{PhD}$ dissertation of the first author, Faculty of Veterinary Medicine, Kazerun Branch, Islamic Azad University, Kazerun.

Authors' contributions

All authors contributed in preparing this article.

\section{Conflicts of interest}

The authors declared no conflict of interest. 
This Page Intentionally Left Blank 


\title{
بررسى مقايسهاى اثرعصاره هيدروالكلى كَل محمدى و تخهم كتان بر فاكتورهاى كبدى موشهاى

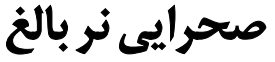

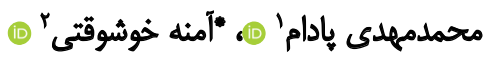 \\ 1. دانشكده داميزشكى، واحد كازرون، دانشعاه آزاد اسلامى، كازرون، ايران.

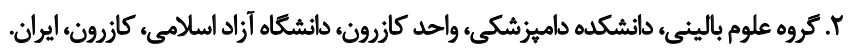

\begin{abstract}
حكSد

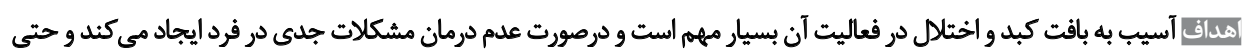

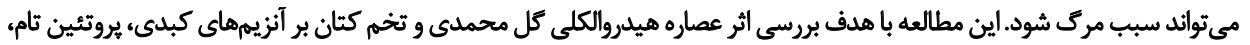

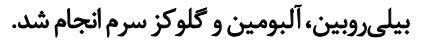

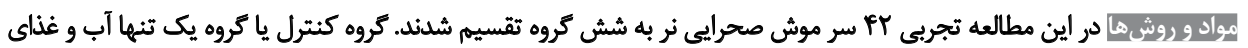

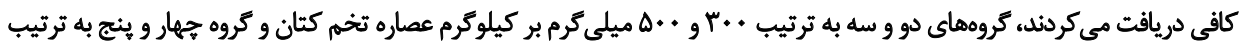

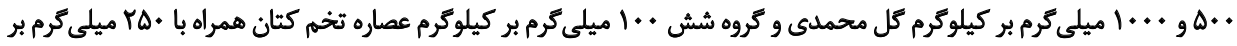

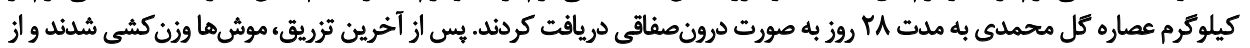

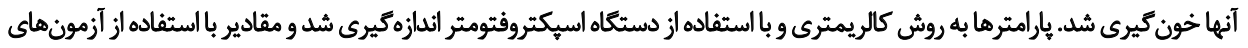

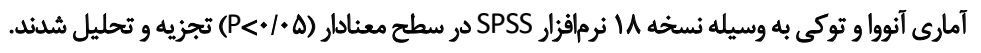

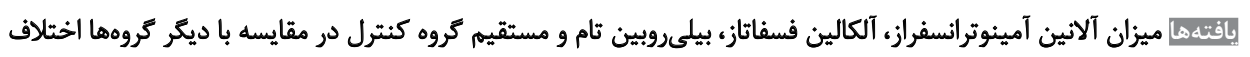

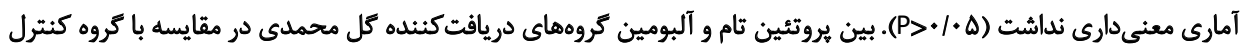

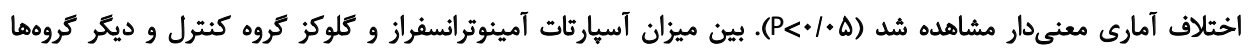

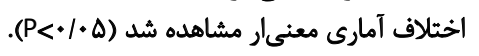

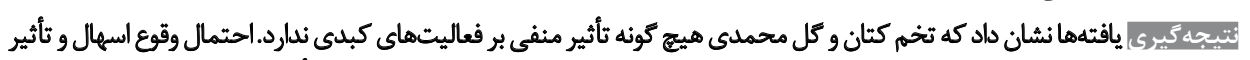

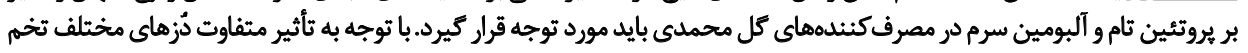

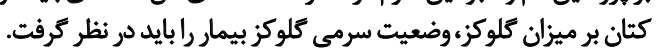

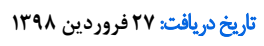

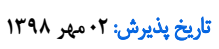

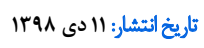

كليدواروها:

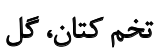

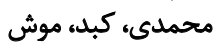
صحرايي محماي،

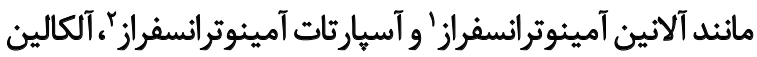

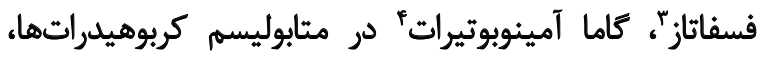

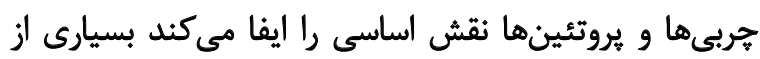

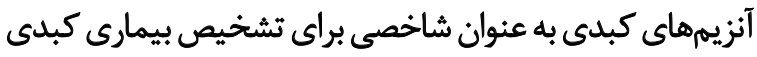

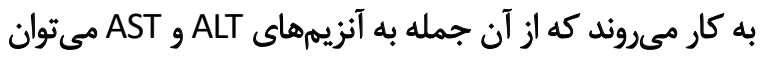

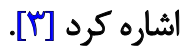

آلبومينه، بروتئين اصلى بِلاسما بوده و عملكرد اصلى آن آن

1. Alanin Amino Transferase (ALT)

2. Aspartate Amino Transferase (AST)

3. Alkaline Phosphatase (ALP)

4. Gama Glutamyl Transferase (GGT)

5. Albumin (Alb)

doง

كبد مسئول اعمال حياتى در بدن است، بيمارى و اختلال در

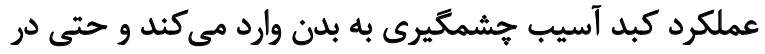

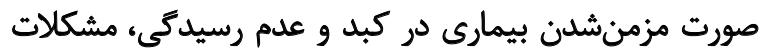

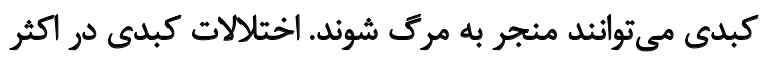

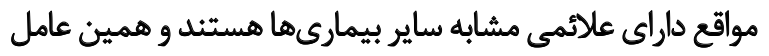

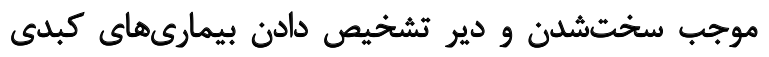

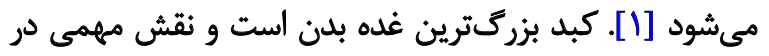

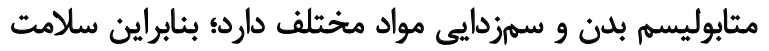

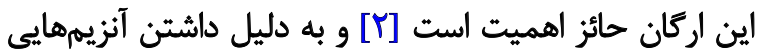

\footnotetext{
* : نويسئده مسئول:

دكتر آمنه خوشوقتى

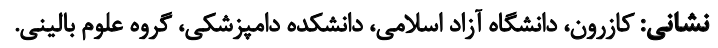
تلفن: يست الكترونيكى: khoshvaghti2004@yahoo.com
} 
نقش مهمى در بهبود عملكرد عروق دارد. استفاده از روغن دانه

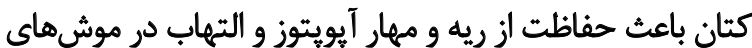

صحرايى مي شود [19]

دانه كتان به دليل خواص آنتىاكسيدانى بالا علاوه بر كاهش

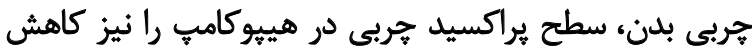

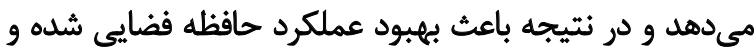

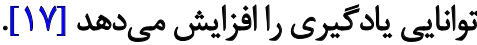

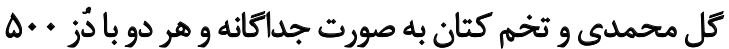

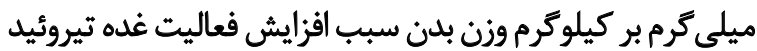
و در نتيجه افزايش ترى يدوتيرونين و ونيدي كاهش

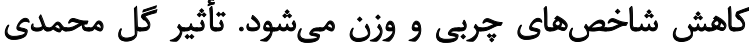

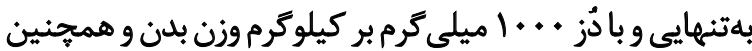

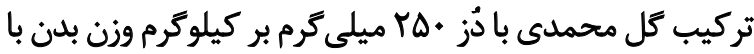

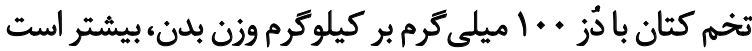

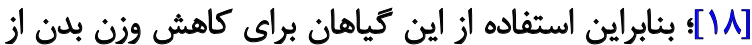

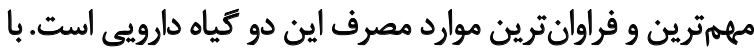

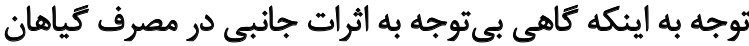

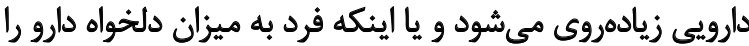

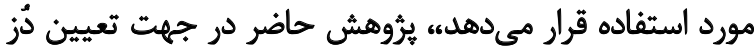

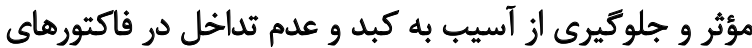

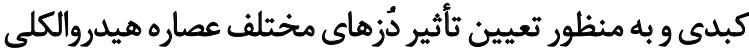

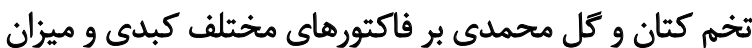

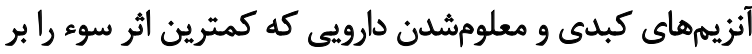

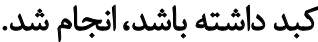

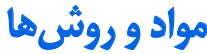

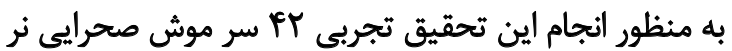

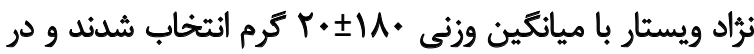

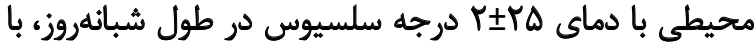

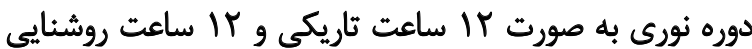

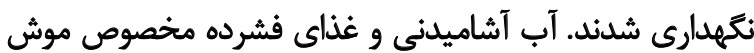

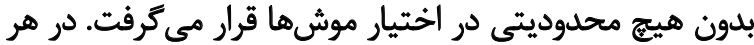

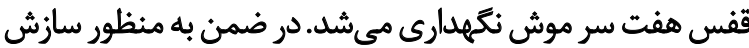

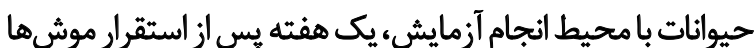

$$
\text { تحقيق در محيط آغاز شد. }
$$

براى تهيه عصاره از روشهاى استاندارد عصارهيرى استفاده

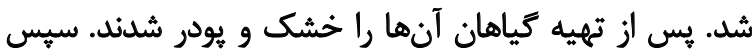

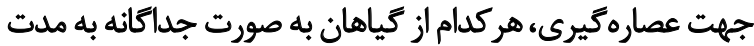

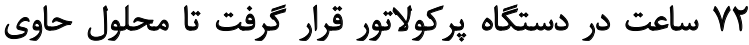

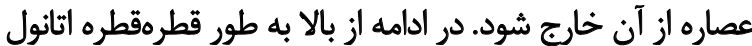

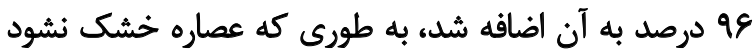

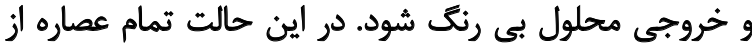

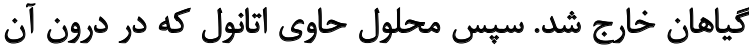

تنظيم فشار اسمزى كلوئيدى خون است [†']. آلكالين فسفاتاز

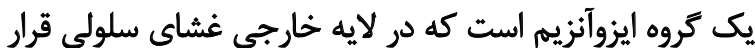

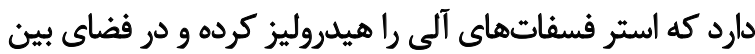

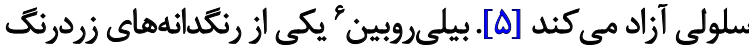

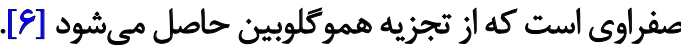
كبد با نقش مههمى كه در متابوليسم و ذخيره كلوكز به صورت

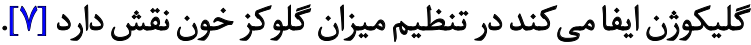
استفاده از كياهان دارويى از زمانهاي قديم رايج بوده و عوارض

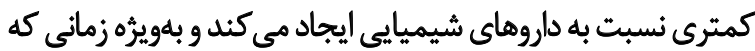

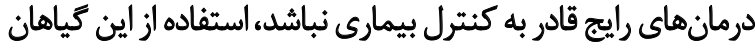

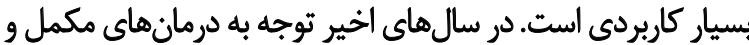

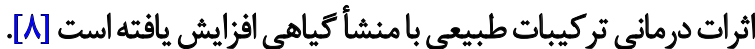

كل محمدى با نام علمى Rosa damascene mill از خانواده

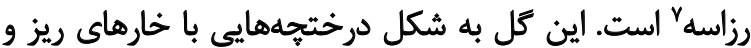

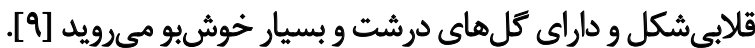

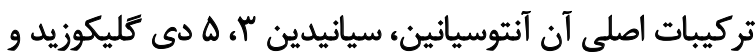

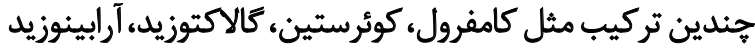

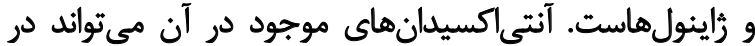

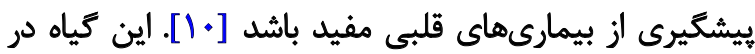

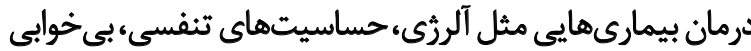

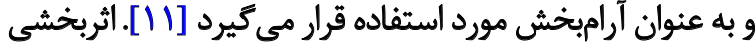

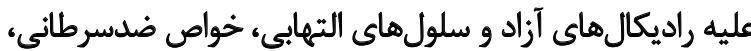

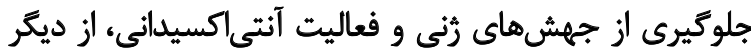

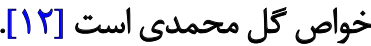

در بروهش ديكرى در مورد كل محمدى به اين نتيجه رسيدند

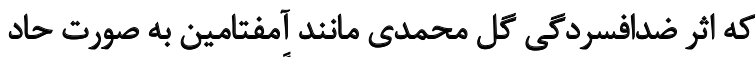

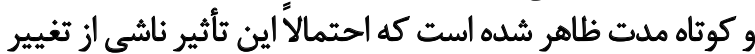

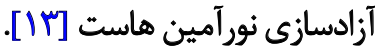

كياه كتان با نام علمى Linum Usitatissumum كياهى

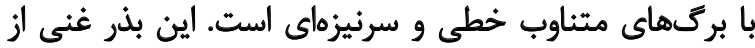

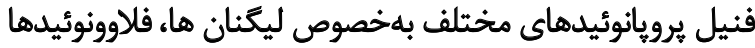

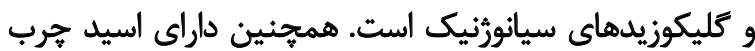

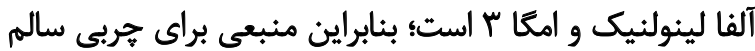

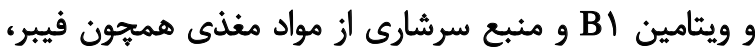

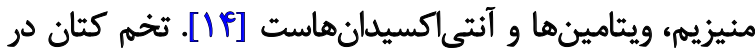
مبتلايان سندرم روده تحريكيذئير، زنان باردار و وافراد باد با تخمدان

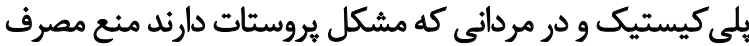

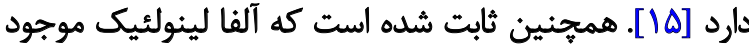

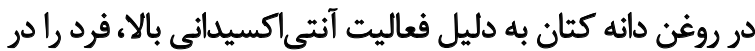

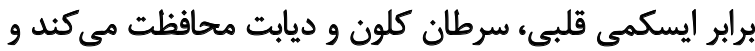

6. Bilirubin (Bili)

7. Rosaceace 


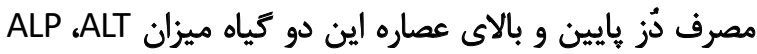

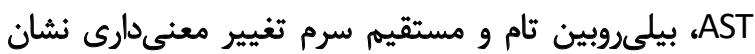

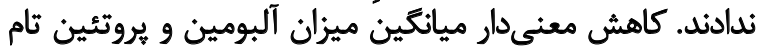

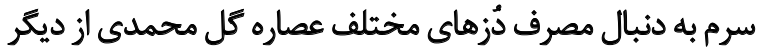
يافتههاى اين تحقيق است.

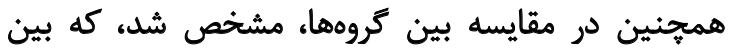

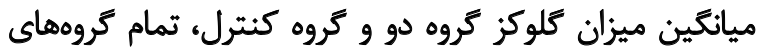

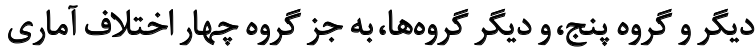

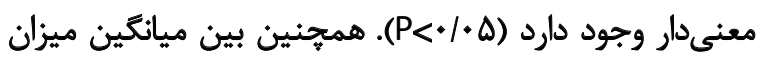

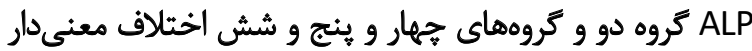

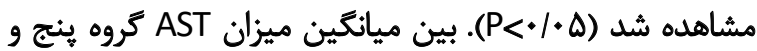

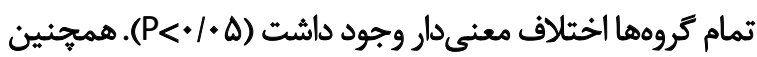

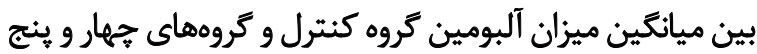

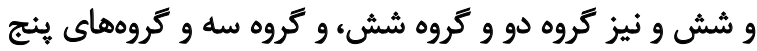

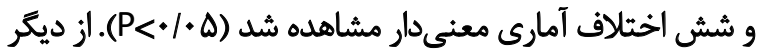

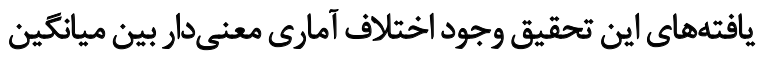

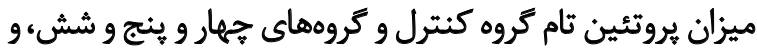

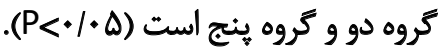

ثب

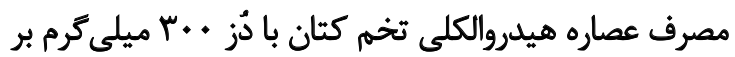

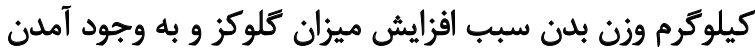

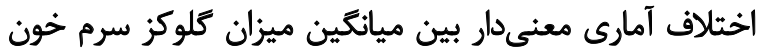

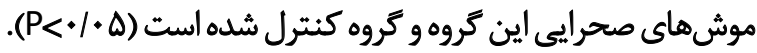

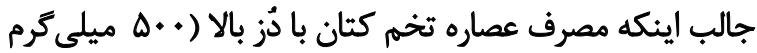

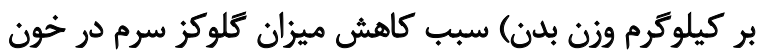

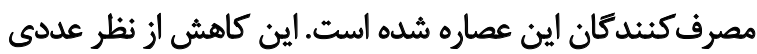

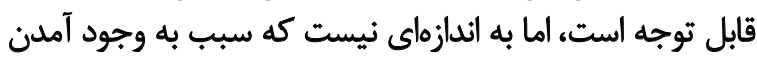

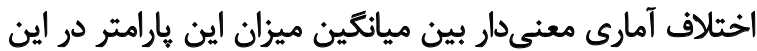

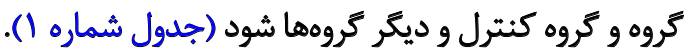

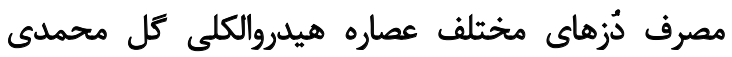

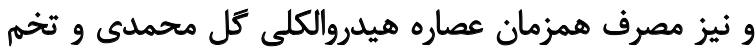

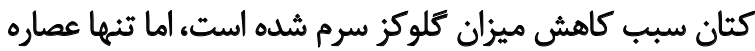

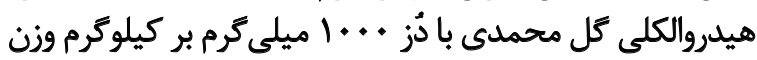

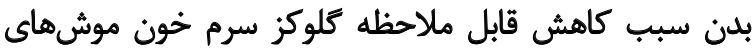

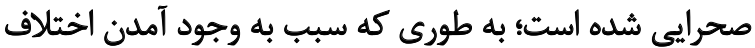

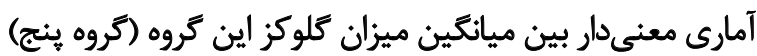

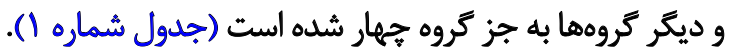
نيكابخت و همكاران در سال |وسا تزارش كردند كه

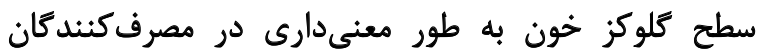

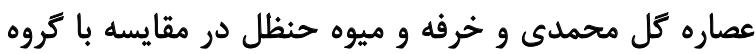

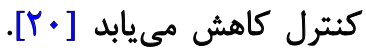

عصاره كياه مدنظر، قرار داشت در دستكاه روثارى قرار گرفت تأنا

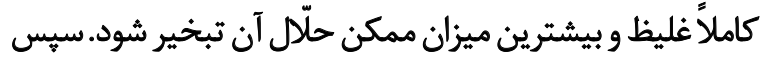

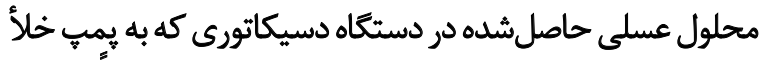

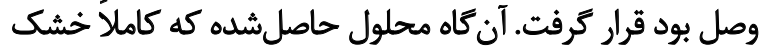

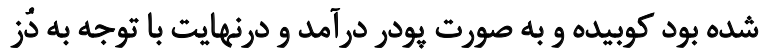

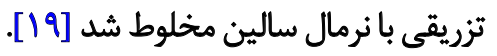

حيوانات به طور تصادفى به شش كروه هفت تئي تقسيم شدند.

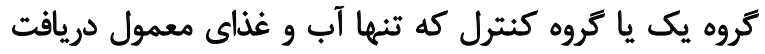

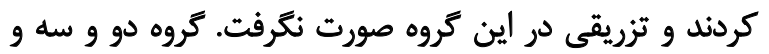

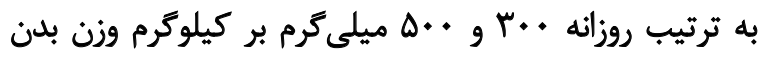

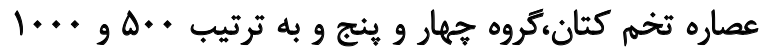

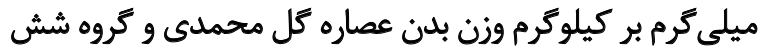

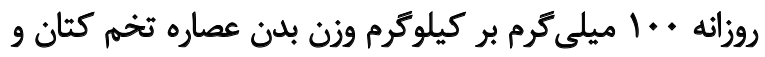

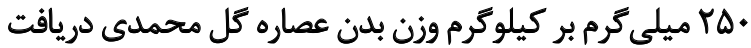

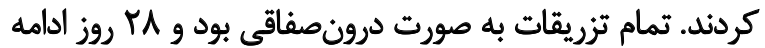

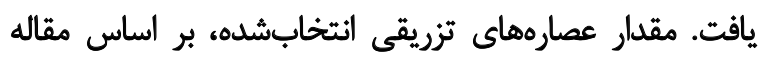

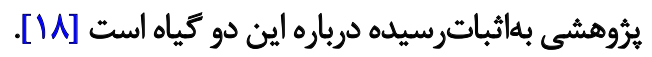

يك روز يس از آخرين تزريق از هريك از موشها دانها در شرايط

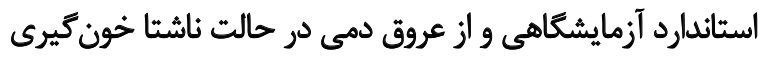

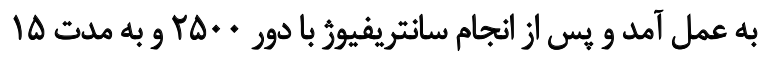

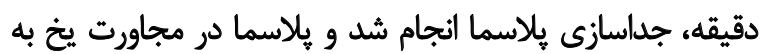

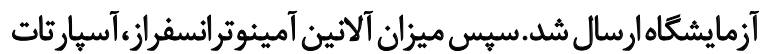

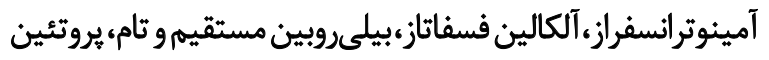

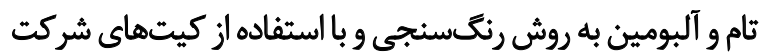

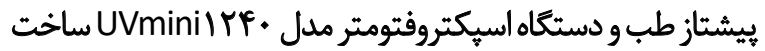

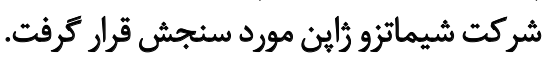

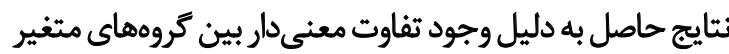

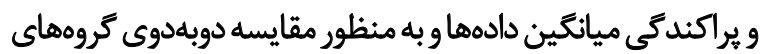

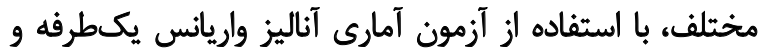

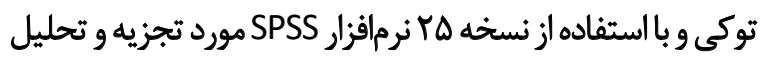

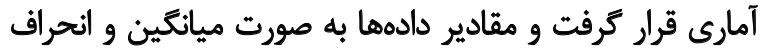

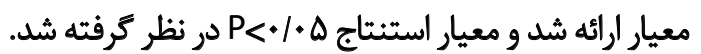

يافتهها

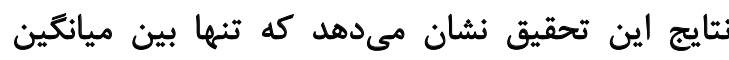

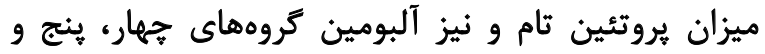

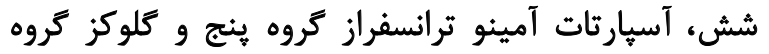

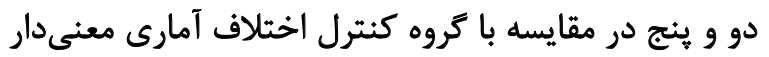

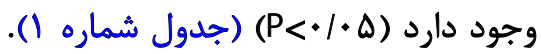
همانطور كه در جدول شماره ا آورده شده است، مصرف

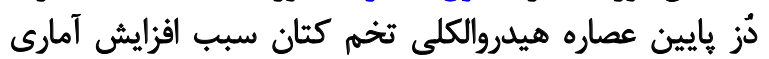

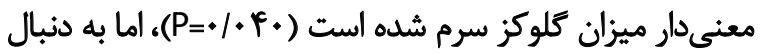


C.:

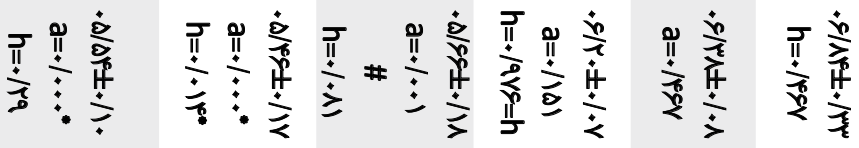

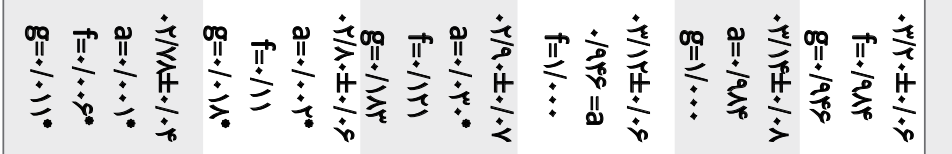

औ.

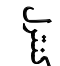

हैं

is

整

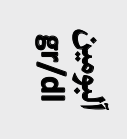

$\frac{0}{\frac{0}{x}}$

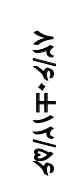

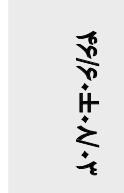

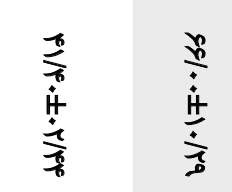

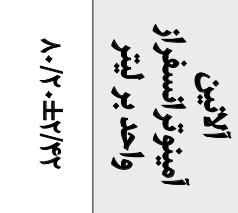

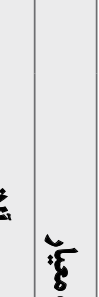

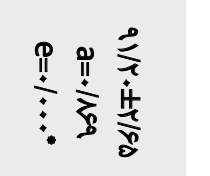

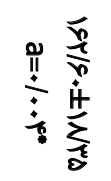

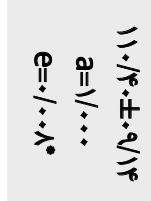

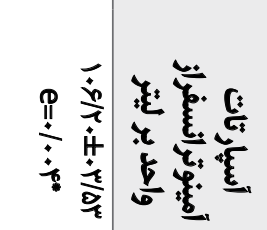

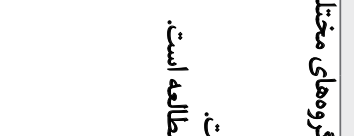

I. 일

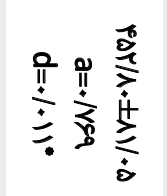

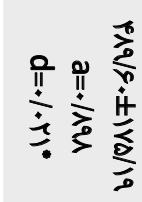

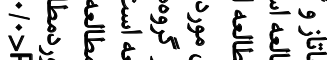

कद

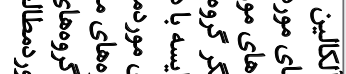

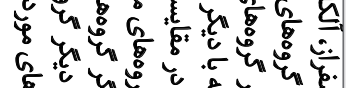

s

w

E.

o

s

$\stackrel{\dot{⿱ 亠 亠}}{+}$

$\stackrel{+}{+}$

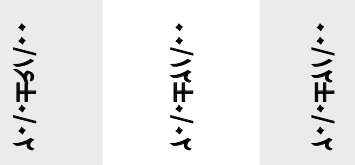

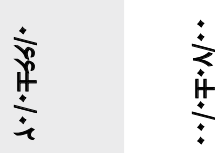

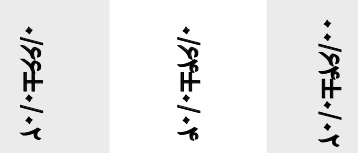

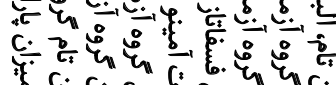

c. is of a

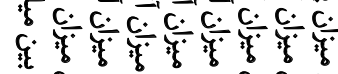

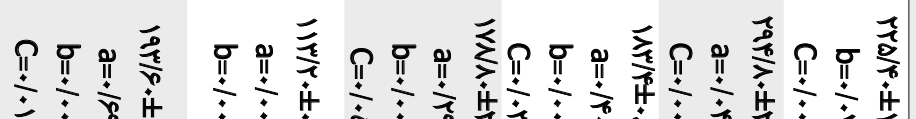

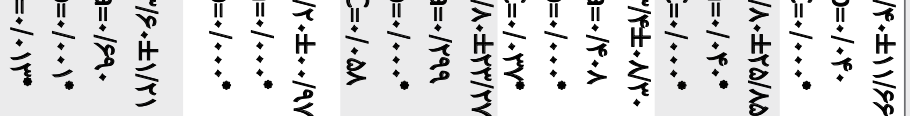

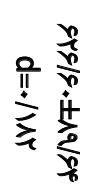

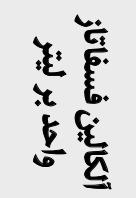

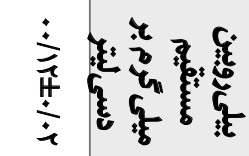

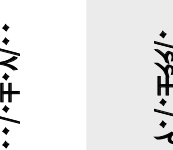

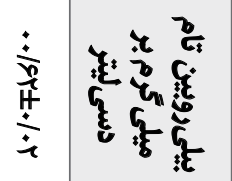

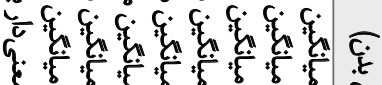

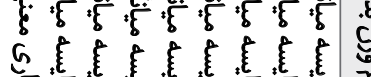

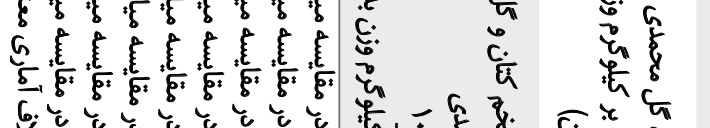

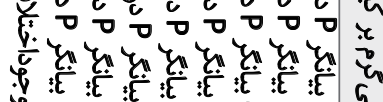

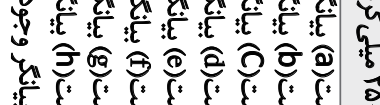

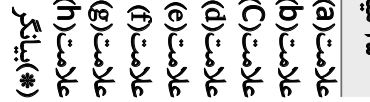
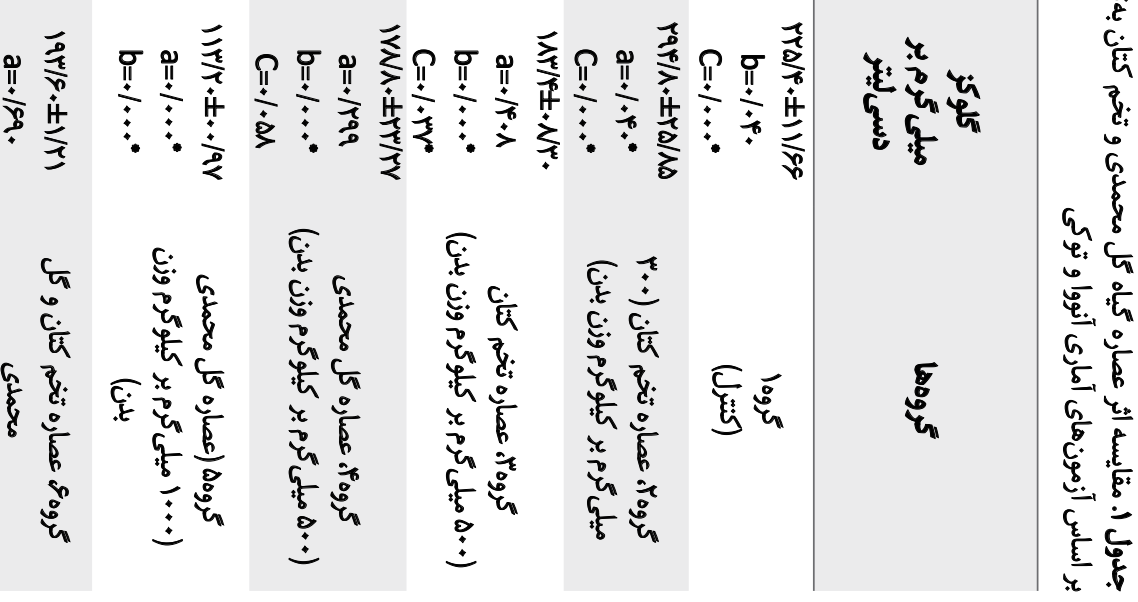
فلاونوئيدهاي اين عصارهها غالب است؛ به طورى كه مصرف اين

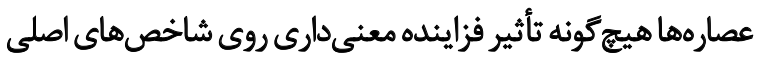

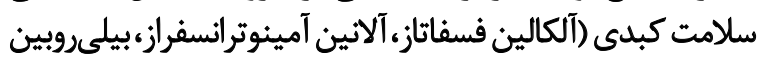

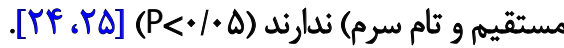

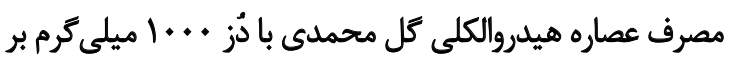

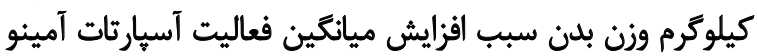

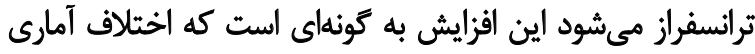

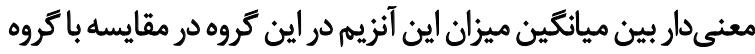

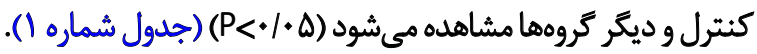

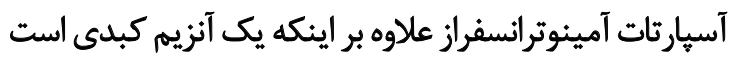

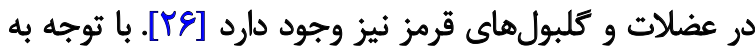

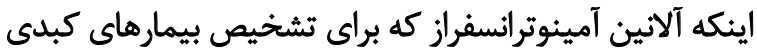

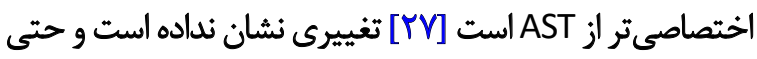

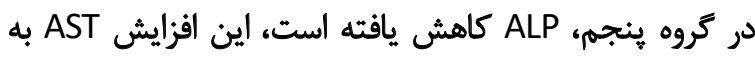

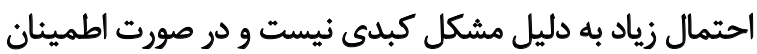

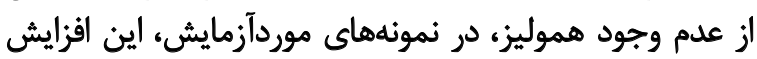

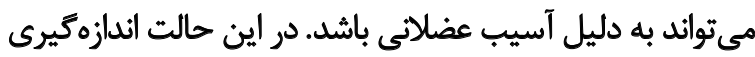

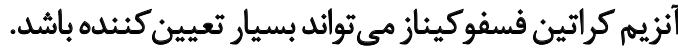

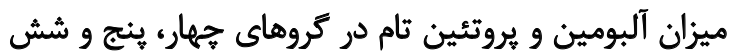

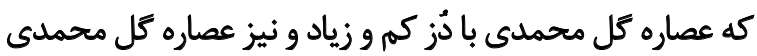

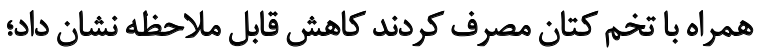

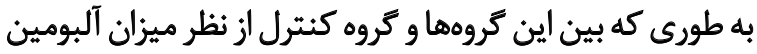

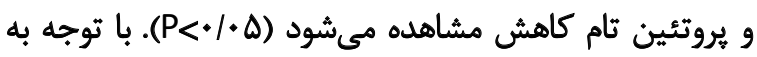

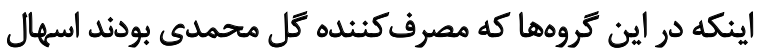

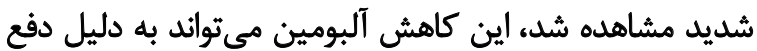

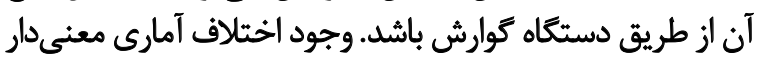

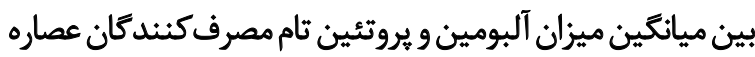

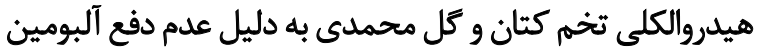

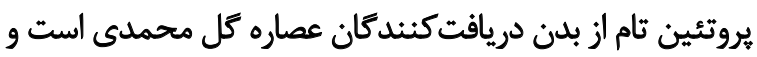

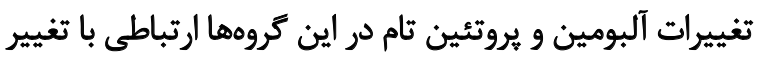
روند سنتز يروتئينها در كبد ندارد.

كلوريزوكا و همكاران در يُروهشى عنوان كردند كه تخمي

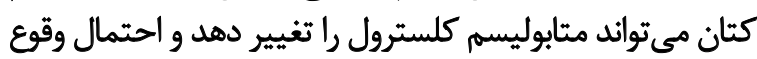

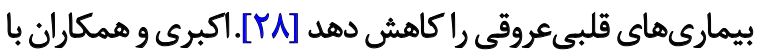

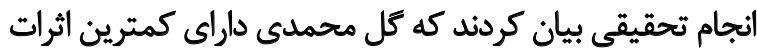

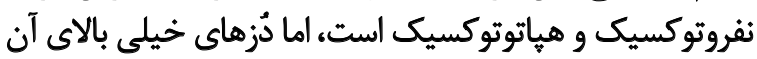

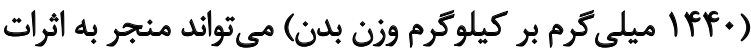

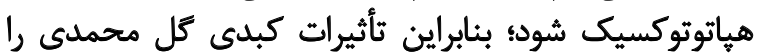

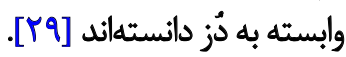

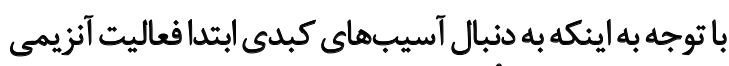

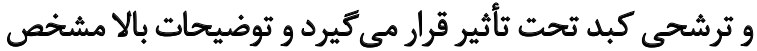

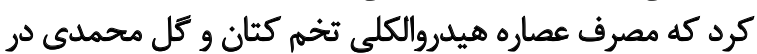

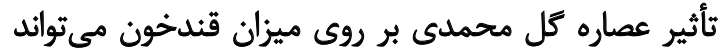

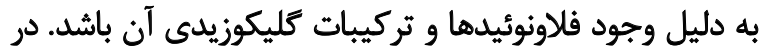

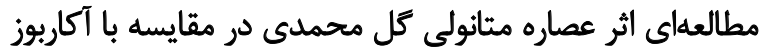

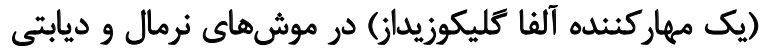

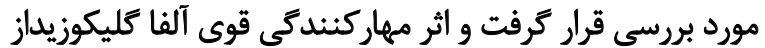

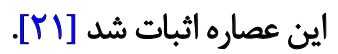

هبه نظر ميرسد تأثير ميزانهاي مختلف تركيبات كليكوزيدي،

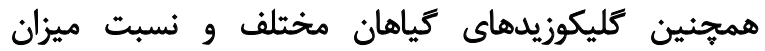

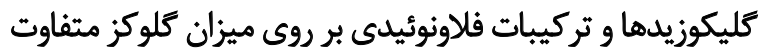

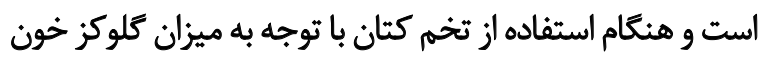

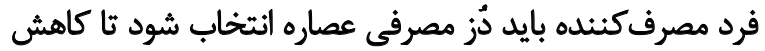

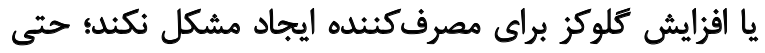

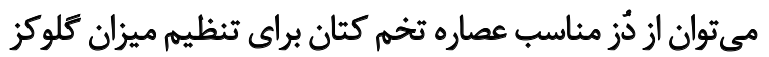

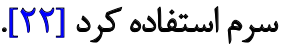

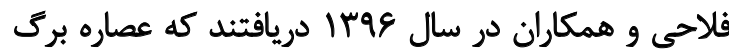

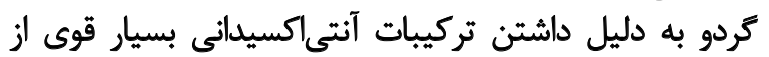

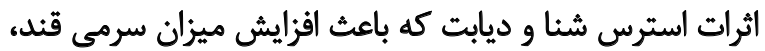

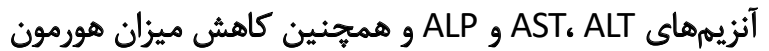

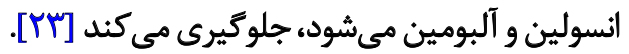

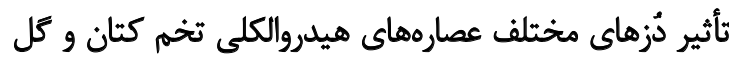

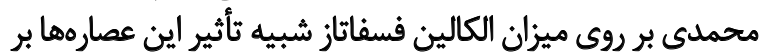

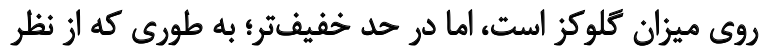

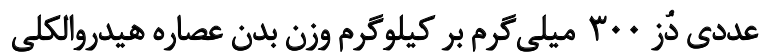

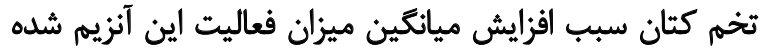

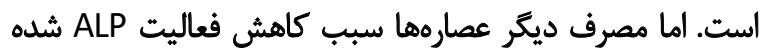

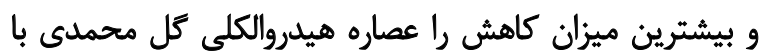

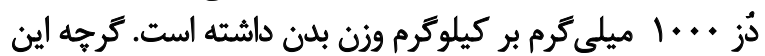

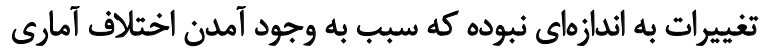

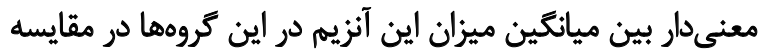

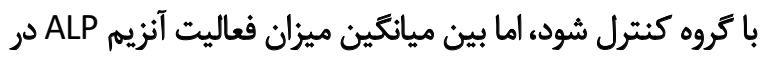

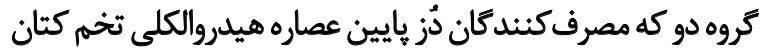

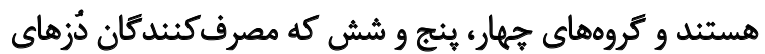

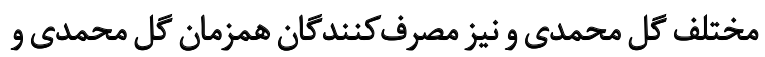

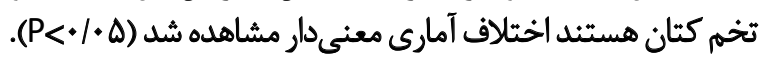

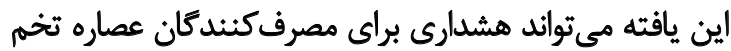

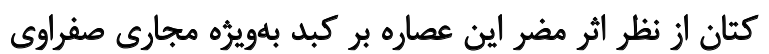

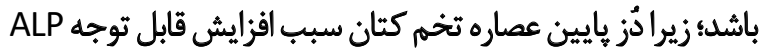

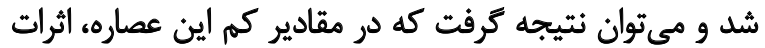

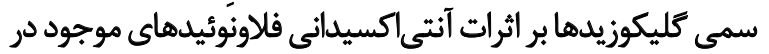

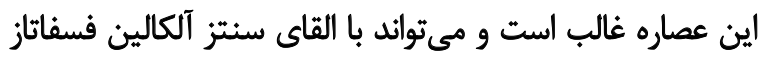

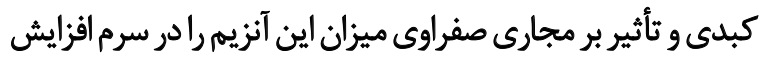

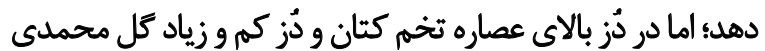
و حتى تركيب عصاره هر دوى اين كياهان، اثر آنتى داكسيدانى 


\section{مشاركت ثويسئدكان}

ايده اصلى، طراحى مطالعه، جمع آورى داده، بازنويسى دستنوشته

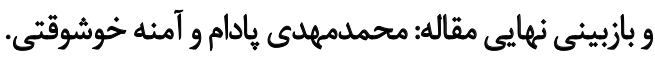

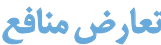

بنابر اظهار نويسندكان اين مقاله هيجگونه تعارض منافع ندارد.

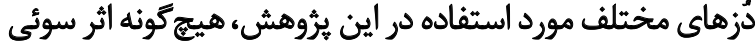

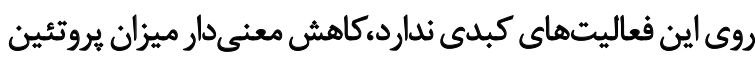

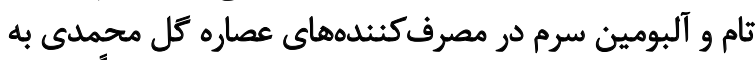

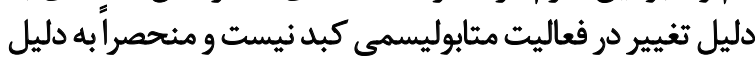

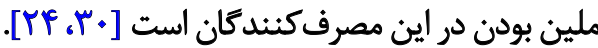
آل نواس و همكاران كزارش كردند كه مصرف روزانه دُز بالاي

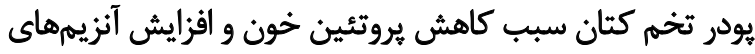

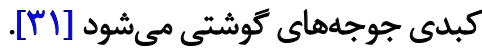

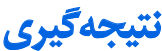

بررسى نتايج تحقيق حاضر نشان داد كه مصرف عصاره

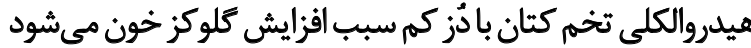

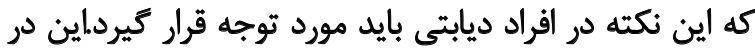

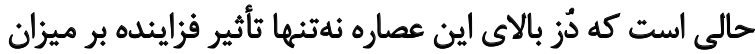

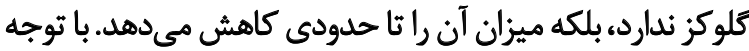

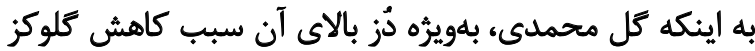

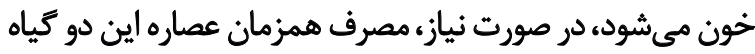

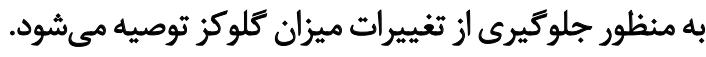

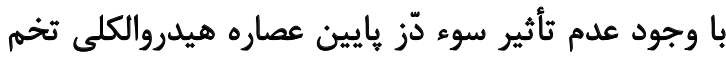

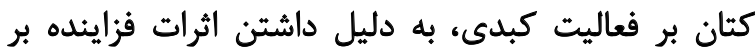

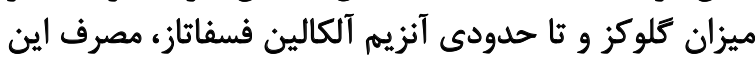
عصاره با دُز يايين توصيه نمئ حدود انزيم الكالين. صرفنظر از تأثير دُز يايين تخمم كتان بر افزايش فعاليت ALP،

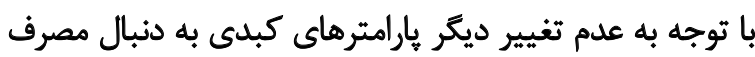

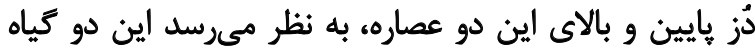

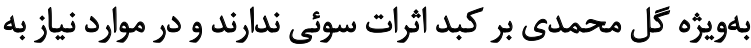

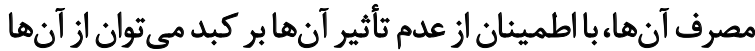

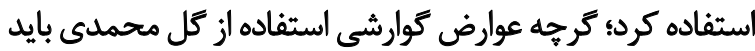

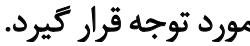
مالاحظات اخلاقى

\section{يميروى الؤ أصول اخلاق بروهش}

اين يثوهش بر اساس قوانين و دستورالعمل كميته اخلاق

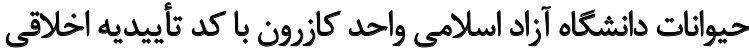

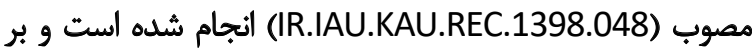
اين اساس اخلاق زيستى و حقوق حيوانات موردمطالعه لحاظ شد.

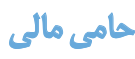

اين مقاله حاصل ياياننامه محمدميهدى يادام، دانشجوى دكثراى

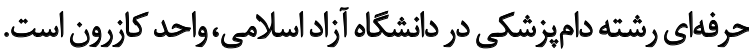




\section{References}

[1] Wen D, Li C, Di H, Liao Y, Liu H. A universal HPLC method for the determination of phenolic acid in compound herbal medicines. Journal of Agricultural and Food Chemistry. 2005; 53(17):6624-9. [DOI:10.1021/jf0511291] [PMID]

[2] Suzuki K, Tanaka M, Watanabe N, Saito Sh, Nonaka H, Miyajima A. p75 neurotrophin receptor is a marker for precursors of stellate cells and portal fibroblasts in mouse fetal liver. Gastroenterology. 2008; 135(1):270-81.e3. [DOI:10.1053/j.gastro.2008.03.075] [PMID]

[3] Żuk M, Kulme A, Dymińska L, Szołtysek K, Prescha A, Hanuza J, et al. Fhavonoid engineering of flax potentiate Its biotechnological application. BMC Bioteehnology. 2011; 11:10. [DOI:10.1186/1472-675011-10] [PMID] [PMCID]

[4] Lichenstein HS, Lyons DE, Wurfel MM, Johnson DA, McGinley MD, Christopher Leidli J, et al. Afamin is a new member of the albumin, alpha-fetoprotein, and vitamin D-binding protein gene family. Journal of Biological Chemistry. 1994; 269(27):18149-54. https://scinapse.io/ papers/1497693870

[5] Kim EE, Wyckoff HW. Reaction mechanism of alkaline phosphatase based on crystal structures: Two-metal ion catalysis. Journal of Molecular Biology. 1991; 218(2):449-64. [DOI:10.1016/0022-2836(91)90724-K]

[6] Lightner DA. Early scientific investigations. In: Lightner DA. Bilirubin: Jekyll and Hyde Pigment of Life, Progress in the Chemistry of Organic Natural Products. Vol. 98. Wien: Springer-Verlag; 2013. p. 9-179. [DOI:10.1007/978-3-7091-1637-1]

[7] Kawai Sh, Mukai T, Mori Sh, Mikami B, Murata K. Hypothesis: Structures, evolution, and ancestor of glucose in the hexokinase family. Journal of Bioscience and Bioengineering. 2005; 99(4):320-30. [DOI:10.1263/jbb.99.320] [PMID]

[8] Middleton Jr E, Kandaswami C. The impact of plant flavonoids on mammalian biology: Implications for immunity, inflammation and canaer. In: Harborne JB, editor. The Flavonoids Advances in Research Since 1986. Vol. 3. Boca Raton: CRC Press; 1994. p. 619-53. https://books.google. com/books?id=SEZdLxbep4IC\&dq

[9] Sadraei H, Asghari G, Emami S. Effect of Rosa damascene mill. flower extract on rat ileum. Research in Pharmaceutical Sciences. 2013; 8(4):277-84. [PMID] [PMCID]

[10] Velioglu YS, Mazza G. Characterization of flavonoids in petals of Rosa damascene by HPLC and spectral analysis. Journal of Agricultural and Food Chemistry. 1991; 39(3):463-7. [DOI:10.1021/jf00003a007]

[11] Loghmani-Khouzani H, Sabzi Fini O, Safari J. Essential oil composition of Rosa damascena mill cultivated in central Iran. Scientia Iranica. 2007; 14(4):316-9. https://iranjournals.nlai.ir/2127/article_517128.html

[12] Crespo ME, Gálvez J, Cruz T, Ocete MA, Zarzuelo A. Anti-inflammatory activity of diosmin and hesperidin in rat colitis induced by TNBS. Planta Medica. 1999; 65(7):651-3. [DOI:10.1055/s-2006-960838] [PMID]

[13] Zarghami M, Farzin D, Bagheri K. [Anti depressant effects of Rosa damascena on laboratory rats (a controlled experimental blind study) (Persian)]. Journal of Mazandaran University of Medical Sciences. 2001; 11(33):27-33. http://jmums.mazums.ac.ir/article-1-59-en.html

[14] Oomah BD, Mazza G. Flaxseed products for disease prevention. In: Mazza G, editor. Functional Foods: Biochemical and Processing Aspects. Lancaster, PA: Technomic Publishing Company; 1998. p. 91-138. https://books.google.com/books?id=01jDyr1EkhEC\&vq

[15] Farahpour MR, Taghikhani H, Habibi M, Zandieh MA. Wound healing activity of flaxseed Linum usitatissimum L. in rats. African Jour- nal of Pharmacy and Pharmacology. 2011; 5(21):2386-9. https:// www.researchgate.net/publication/269676752

[16] Razi SS, Latif MJ, Li X, Afthinos JN, Ippagunta N, Schwartz G, et al. Dietary flaxseed protects against lung ischemia reperfusion injury via inhibition of apoptosis and inflammation in a murine model. Journal of Surgical Research. 2011; 171(1):e113-e21. [DOI:10.1016/j.jss.2011.06.017]

[17] Fernandes FS, de Souza AS, do Carmo MGT, Boaventura GT. Maternal intake of flaxseed-based diet (Linum usitatissimum) on hippocampus fatty acid profile: Implications for growth, locomotor activity and spatial memory. Nutrition. 2011; 27(10):1040-7. [DOI:10.1016/j.nut.2010.11.001] [PMID]

[18] Adibi A, Khoshvaghti A. [A comparative study on the effects of Linum usitatissimum and Rosa domascena hydro-alcoholic extracts on body weight, thyroid hormones, and lipids profiles in rats (Persian)]. Journal of Shahid Sadoughi University of Medical Sciences. 2019; 27(2):1290-301. [DOI:10.18502/ssu.v27i2.1048]

[19] Enayati N, Ghafarzadegan R, Hajiaghaee R, Vazirian M. [Comparison of different methods in sennoside extraction from Senna alexandrina (Persian)]. Journal of Medicinal Plants. 2017; 4(64):160-9. http://jmp.ir/article-1-1917-en.html

[20] Nikbakht MR, Gheatasi I. [Evaluation of the effect of hydroalcoholic extract of Citrullus colocynthis in normoglycemic and Streptozocine (STZ) induced diabetic male rats (Persian)]. Armaghan-e Danesh. 2006; 11(2):63-71. http://armaghanj.yums. ac.ir/article-1-730-fa.html

[21] Hashem Dabaghian F, Kamalinejad M, Shojaii A, Abdollahi Fard M, Ghushegir SA. [Review of antidiabetic plants in Iranian traditional medicine and their efficacy (Persian)]. Journal of Medicinal Plants. 2012; 1(41-S8 Suppl 8):1-11. http://jmp.ir/article-1-466-en.html

[22] Nazem Jahan MA. [Gharabadine azam (the great qarabadin) (Persian)]. Tehran: Research Institute for Islamic \& Complementary Medicine; 2003. http://opac.nlai.ir/opac-prod/bibliographic/2128652

[23] Fallahi M, Hosseini SE. [Effects of walnut leaf hydro-alcoholic extract by forced swimming stress on serum levels of glucose, insulin and liver parameters in adult male rats' diabetic (Persian)]. Journal of Babol University of Medical Sciences. 2017; 19(5):4752. [DOI:10.22088/jbums.19.5.47]

[24] Ciolino HP, Daschner PJ, Yeh GC. Dietary flavonols quercetin and kaempferol are ligands of the aryl hydrocarbon receptor that affect CYP1A1 transcription differentially. Biochemical Journal. 1999; 340(Pt 3):715-22. [DOI:10.1042/bj3400715] [PMID] [PMCID]

[25] Bhatia AL, Manda K, Patni Sh, Sharma AL. Prophylactic action of linseed (Linum usitatissimum) oil against cyclo-phosphamideinduced oxidative stress in mouse brain. Journal of Medicinal Food. 2006; 9(2):261-4. [DOI:10.1089/jmf.2006.9.261] [PMID]

[26] Almo SC, Smith DL, Danishefsky AT, Ringe D. The structural basis for the altered substrate specificity of the R292D active site mutant of aspartate aminotransferase from E.coli. Protein Engineering, Design and Selection. 1994; 7(3):405-12. [DOI:10.1093/ protein/7.3.405] [PMID]

[27] Karmen A, Wróblewski F, LaDue JS. Transaminase activity in human blood. The Journal of Clinical Investigation. 1955; 34(1):126-33. [DOI:10.1172/JCI103055] [PMID] [PMCID]

[28] Króliczewska B, Miśta D, Króliczewski J, Zawadzki W, Kubaszewski $R$, Wincewicz $E$, et al. A new genotype of flax (Linum usitatissimum L.) with decreased susceptibility to fat oxidation: Consequences to hematological and biochemical profiles of 
blood indices. Journal of the Science of Food and Agriculture. 2017; 97(1):165-71. [DOI:10.1002/jsfa.7705] [PMID]

[29] Akbari M, Kazerani HR, Kamrani A, Mohri M. A preliminary study on some potential toxic effects of Rosa damascena mill. Iranian Journal of Veterinary Research. 2013; 14(3):232-6. [DOI:10.22099/IJVR.2013.1686]

[30] Özkan G, Sagdiç O, Baydar NG, Baydar H. Antioxidant and antibacterial activities of Rosa damascena flower extracts. Food Science and Technology International. 2004; 10(4):277-81. [DOI:10.1177/1082013204045882]

[31] Al-Nawass KJ. Effect of different levels of golden flaxseed (Linum usitatissimum L.) powder on some blood biochemical parameters in male and female broiler. Research Opinions in Animal and Veterinary Sciences. 2015; 5(11):425-8. https://www. researchgate.net/publication/331326995 
This Page Intentionally Left Blank 